\title{
Electron kinetics in liquid water excited by a femtosecond VUV laser pulse
}

\author{
Klaus Huthmacher ${ }^{1}$, Nikita Medvedev ${ }^{2}$ and Bärbel Rethfeld ${ }^{1}$ \\ ${ }^{1}$ Department of Physics and OPTIMAS Research Center, \\ Technical University of Kaiserslautern, Germany \\ ${ }^{2}$ Center for Free-Electron Laser Science, DESY, Hamburg, Germany
}

\begin{abstract}
We model numerically the interaction of an ultrashort VUV laser pulse (FWHM = 10 fs, photon energy of $100 \mathrm{eV})$ with liquid water.

The incident laser photons interact with water by ionizing water molecules and creating free electrons. These excited electrons are elastically scattered by water molecules and are able to produce secondary electrons via ionization. To track each free electron and its collisions event by event, we use the Monte Carlo method similar to (N. Medvedev and B. Rethfeld, Transient dynamics of the electronic subsystem of semiconductors irradiated with an ultrashort vacuum ultraviolet laser pulse, New Journal of Physics, Vol. 12, p. 073037 (2010)). This approach allows us to describe the transient non-equilibrium behaviour of excited electrons on femtosecond time scales.

We present transient electron energy distributions and a time resolved energy transfer, i.e.: the changing kinetic energy of excited electrons, the increase of the energy of holes, and excitation of water molecules via elastic collisions. We compare results obtained with different models for the energy levels in liquid water: either assuming dense water vapour or an amorphous semiconductor with a band gap.
\end{abstract}

Keywords: FLASH, femtosecond laser, ultrashort laser, liquid Water, Monte Carlo

\section{INTRODUCTION}

The free electron laser in Hamburg (FLASH) is able to produce ultrashort laser pulses in the extreme ultraviolet (EUV) spectral range [1]. We investigate the electron dynamics of liquid water irradiated by an ultrashort laser pulse with the photon energy and time duration possible with FLASH. The electron dynamics is described on a femtosecond time scale with the Monte Carlo Method (MC) similar to Ref. [2, 3]. We take into account impact ionization, elastic scattering and two models for dissociative recombination.

As results we present time resolved differential and integrated energy transfer rates as well as energy resolved electron densities. We discuss the difference in both our models of the dissociative recombination and show a first approach of describing liquid water as dense water vapour with a density of states. Finally, we present an application of our model to a thin layer of ice.

\section{MONTE CARLO MODEL}

\subsection{Photon absorption}

At first we have to model the photon absorption. We need to know how many photons are absorbed and assume a Gaussian temporal distribution. The number of photons is calculated from the given flux density, average absorption depth [4] and a given simulated control volume, e.g. $10^{3} \mathrm{~nm}^{3}$. We assume that the time resolved intensity $I(t)$ has a Gaussian shape for a laser pulse with a time duration of $\tau=25$ fs and FWHM $=10 \mathrm{fs}$

$$
I(t) \propto e^{-\frac{1}{2} \frac{(t-\mu)^{2}}{\sigma^{2}}}
$$

E-mail: huthmacher@physik.uni-kl.de

Damage to VUV, EUV, and X-ray Optics IV; and EUV and X-ray Optics: Synergy between Laboratory and Space III, edited by René Hudec, Libor Juha, Saša Bajt, Ladislav Pina, Richard London, Proc. of SPIE Vol. 8777, 87770S . (C) 2013 SPIE · CCC code: 0277-786X/13/\$18 - doi: 10.1117/12.2020451 
with the mean $\mu=\tau / 2$. We acquire the variance $\sigma^{2}$ via the correspondence $\mathrm{FWHM}=2 \sqrt{2 \ln (2)} \sigma$. We want to come up with a random variable $t$, which determines the time when a photon is absorbed during laser irradiation, which is distributed according to the intensity profile $I(t)$. This can be achieved via the Inversion Method [5] which leads to the well established Box-Muller-Method [5]

$$
t=\mu+\sigma \sqrt{-2 \ln \left(u_{1}\right)} \cdot \cos \left(2 \pi u_{2}\right) \quad u_{1}, u_{2} \in(0,1),
$$

where $u_{1}$ and $u_{2}$ are two uniformly distributed random numbers. This enables the concept of the Monte Carlo Method (MC) to simulate a time resolved photon absorption on a femtosecond time scale.

For the photon absorption we take into account four distinctive molecule orbitals for water at $I_{1}=12.6 \mathrm{eV}$, $I_{2}=14.7 \mathrm{eV}, I_{3}=18.4 \mathrm{eV}$ and $I_{4}=32.2 \mathrm{eV}[6]$. Electrons, which are created due to photon absorption, start with a kinetic energy of $T=E_{p h}-I_{i}=100 \mathrm{eV}-I_{i}$. We treat all four binding energies $I_{i}$ as equally probable and sample them with an according discrete random variable.

\subsection{Electron dynamics}

Our simulation of electron dynamics treats each electron individually which means that we calculate for each electron the time until the next interaction occurs. The time evolution is simulated in fixed time steps, here $0.1 \mathrm{fs}$. According to the Monte Carlo Method we determine for each time step whether the electron performs a collision or not.

To calculate the average mean free path for the electron between two collisions with a water molecule, we take into account the cross sections of the involved interactions, namely impact ionization, elastic scattering and dissociative recombination. Together with the number density for liquid water $n_{\mathrm{w}}=3 \times 10^{22} \mathrm{~cm}^{-3}$, we calculate the mean free path $\lambda_{0}$ based on the given formula [7]

$$
\lambda_{0}=\frac{1}{n_{\mathrm{w}} \cdot\left(\sigma_{\mathrm{io}}+\sigma_{\mathrm{el}}+\sigma_{\mathrm{dr}}\right)},
$$

where $\sigma_{\mathrm{io}}$ is the energy dependent cross section of ionization, $\sigma_{\mathrm{el}}$ the cross section for elastic scattering and $\sigma_{\mathrm{dr}}$ the cross section for dissociative recombination.

Based on the Monte Carlo Method we have to sample the mean free path (to get a realized length until the next collision occurs) with a uniformly distributed random number $u$

$$
\lambda=-\lambda_{0} \ln (u) \quad \text { with } u \in(0,1) .
$$

Furthermore we derive the velocity of the electron classically from its kinetic energy and can thus calculate the time it takes until another collision occurs.

If interaction takes place we have to decide which kind of interaction. This decision is based on the involved cross sections of the interactions. Since we have only a uniformly distributed random number $u \in(0,1)$ we have to weight the cross sections to come up with a probability between 0 and 1 :

$$
\left(0, \frac{\sigma_{\mathrm{el}}}{\sigma_{\mathrm{total}}}\right),\left[\frac{\sigma_{\mathrm{el}}}{\sigma_{\text {total }}}, \frac{\sigma_{\mathrm{el}}+\sigma_{\mathrm{io}}}{\sigma_{\text {total }}}\right) \text { and }\left[\frac{\sigma_{\mathrm{el}}+\sigma_{\mathrm{io}}}{\sigma_{\text {total }}}, 1\right)
$$

where $\sigma_{\text {total }}=\sigma_{\mathrm{el}}+\sigma_{\mathrm{io}}+\sigma_{\mathrm{dr}}$.

The interactions of elastic scattering and impact ionization lead to energy transfer. In the first case energy is transferred from the electron to a water molecule and second from electron to a newly created electron. We treat the collision between two particles with mass $m_{1}$ and $m_{2}$ classically via the formula:

$$
\Delta E_{\max }=4 \frac{m_{1} \cdot m_{2}}{\left(m_{1}+m_{2}\right)^{2}} T,
$$

where $T$ is the kinetic energy of the incident particle, in our case an electron. Particle two is assumed to be at rest. 
In the case of elastic scattering, we set without loss of generality $m_{1}=m_{\text {electron }}$ and $m_{2}=m_{\text {water }}$. Since $m_{\text {electron }}<<m_{\text {water }}$ holds true we end up with the approximation

$$
\Delta E_{\max }^{\mathrm{el}} \approx 4 \frac{m_{\text {electron }}}{m_{\text {water }}} T .
$$

Due to the huge difference in the mass of electrons and water molecules only a small amount of energy is transferred.

In the case of ionization, the kinetic energy of the incident electron has to overcome the binding energy first. This leads to a possible maximal energy transfer of $\Delta E_{\max }^{\mathrm{io}}=1 / 2\left(T-I_{i}\right)$. The coefficient $1 / 2$ is needed since the electrons are indistinguishable and by definition the slower one is the newly created one.

In both cases, elastic scattering and impact ionization, we assume that the transferred energy is uniformly distributed and we determine the transferred energy by using a uniformly distributed random number $u$

$$
\Delta E=u \cdot \Delta E_{\max }^{\mathrm{el}, \mathrm{io}} \quad u \in(0,1) .
$$

\subsection{Cross Sections}

As shown in equation (3) we need the cross sections of the included processes to simulate the time evolution of the electronic system. First, the cross section for impact ionization is taken from Ref. [6], which was already used in Ref. [8]. The cross section for elastic scattering is fitted from the data in Ref. [7].

The dissociative recombination leads to the neutral parts of the water molecule due to the recombination of single ionized water molecule $\mathrm{H}_{2} \mathrm{O}^{+}$and an electron and the following dissociative process.

The cross section $\sigma_{\mathrm{dr}}$ for the dissociative recombination needs a more detailed description. We distinguish between two methods of including the dissociative recombination in our model. The first method is a numerical tool to come up with an energetic lower boundary. If an electron has a kinetic energy of lower than $0.01 \mathrm{eV}$ it is removed from the electronic system. In that case, the cross section does not contribute to the mean free path as shown in equation (3) and is set to zero. We call that method an artificial dissociative recombination.

In the second case, we use the energy dependent cross section $\sigma_{\mathrm{dr}}(T)$ from Ref. [9]. We fitted the data shown in Figure 4 in that paper and also correlate the cross section to the number of single ionized water molecules.

\section{RESULTS}

\subsection{Influence of dissociative Recombination}

As a first result Figure 1 shows the energy resolved electron density at distinctive time steps, namely $10 \mathrm{fs}, 30 \mathrm{fs}$, $100 \mathrm{fs}$ and $1000 \mathrm{fs}$. The corresponding simulation uses the artificial cross section for dissociative recombination as described in the previous section.

The red curve shows four little peaks, which represent the four distinctive free electron energies $T=E_{\mathrm{ph}}-I_{i}$. This is due to the fact, that free electrons are created via photon absorption which did not interact yet during this time step at $10 \mathrm{fs}$. Until the laser pulse is gone, e.g. after $25 \mathrm{fs}$, further free electrons are created due to photon absorption. In addition to that, electrons can also create secondary electrons via impact ionization. Both processes lead to a rapid increase in the electron density, as the blue curve shows at $30 \mathrm{fs}$.

The electrons transfer their kinetic energy via elastic scattering and ionization to water molecules or secondary electrons, respectively. The blue and black curve in Figure 1 show a sharp bend at roughly $13 \mathrm{eV}$. The lowest binding energy is $12.6 \mathrm{eV}$. Electrons with a kinetic energy below this threshold cannot create further secondary electrons and are left to transfer their energy via elastic scattering. After $1000 \mathrm{fs}$, the green curve shows that the majority of the electrons posses a very small amount of kinetic energy. Multiple elastic scattering processes, with each a small energy transfer according to equation (7), have shifted the maximum kinetic energy of electrons to lower values.

Figure 2 shows the results of the same simulation but with an energy dependent dissociative cross section according to Ref. [9], see previous section. This Figure shows a drastic change in the course of the curves and also in the total electron density. In contrast to Figure 1, electrons with a kinetic energy higher than $0.01 \mathrm{eV}$ can be removed due to dissociative recombination. As can be seen, after $1000 \mathrm{fs}$ nearly all electrons are removed from the electronic system. 


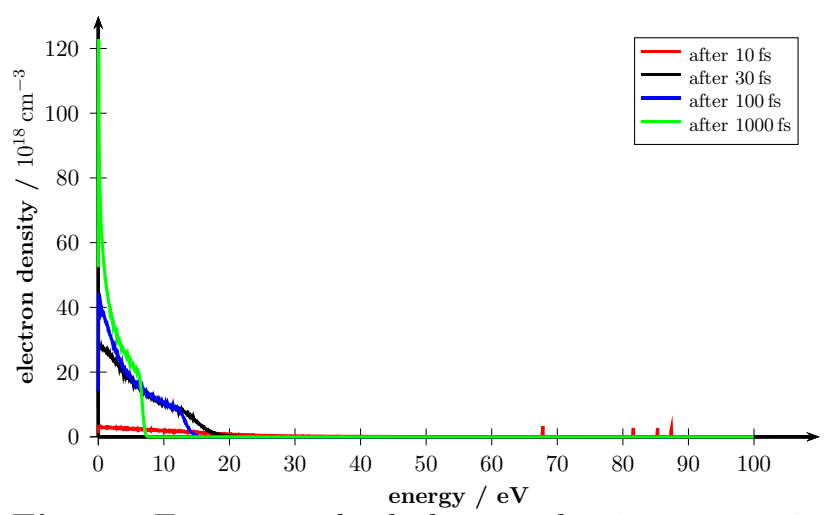

Fig. 1: Energy resolved electron density at certain time steps, calculated with artificial dissociative recombination.

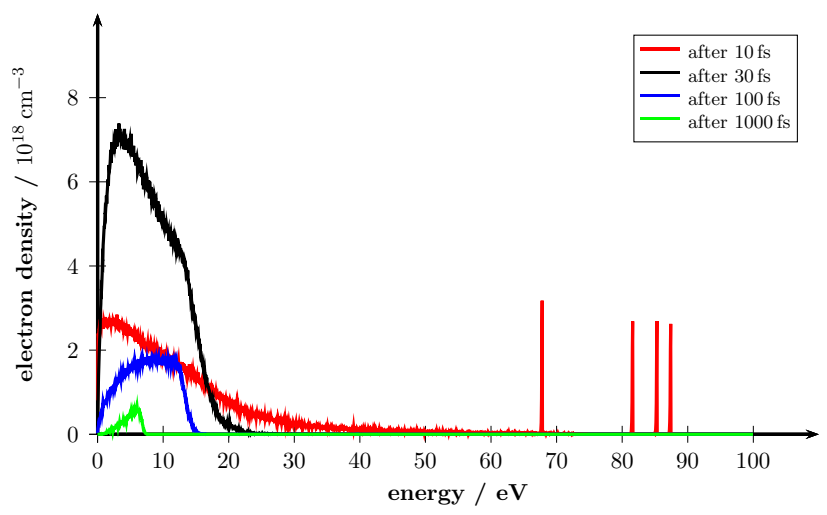

Fig. 2: Energy resolved electron density at certain time steps, calculated with dissociative recombination from data set [9].

In Figure 3 and 4 it can be seen where the photon energy is transferred to. Figure 3 corresponds again to the simulation with the so called artificial dissociative recombination while 4 accounts for an energy dependent cross section according to Ref. [9].

Figure 3 shows the four channels where the energy, brought into the system via photon absorption, is transferred to. A huge amount is stored in the holes (blue curve) by photon absorption and impact ionization, which is lost for the electronic system. As can be seen, the two ionization processes occur completely during the laser irradiation. A significant amount of energy is transferred to the water molecules via elastic scattering (red curve). This is important since we consider this increase of the molecule energy as heating up the water sample. This is a very slow process and happens on a pico second time scale. Finally the kinetic energy of the free electrons decreases (green curve) due to impact ionization (fast), elastic scattering (slow) and the artificial dissociative recombination (slow). The latter channel is shown as a black curve.

Figure 4 shows the energy channels with an energy dependent cross section for dissociative recombination which accounts for the degree of ionization. Note that only the first pico second is shown since here the processes take place. As can be seen the course of the curves changes drastically. The process of dissociative recombination dominates the flow of energy. At first, dissociative recombination removes the kinetic energy of the free electrons out of the electronic system, since it removes them and second, it also takes out the hole energy, due to the recombination of $\mathrm{H}_{2} \mathrm{O}^{+}$and $\mathrm{e}^{-}$. As a consequence only a fraction of the absorbed photon energy is transferred via elastic scattering to the water molecules.

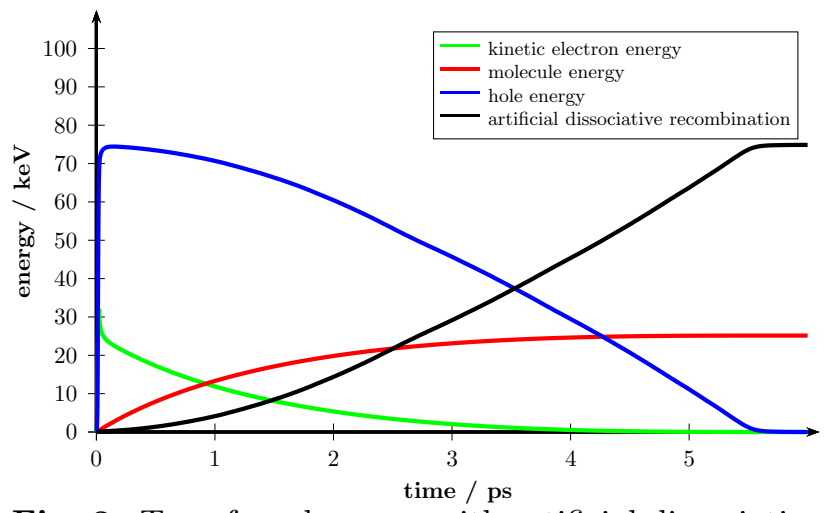

Fig. 3: Transferred energy with artificial dissociative recombination

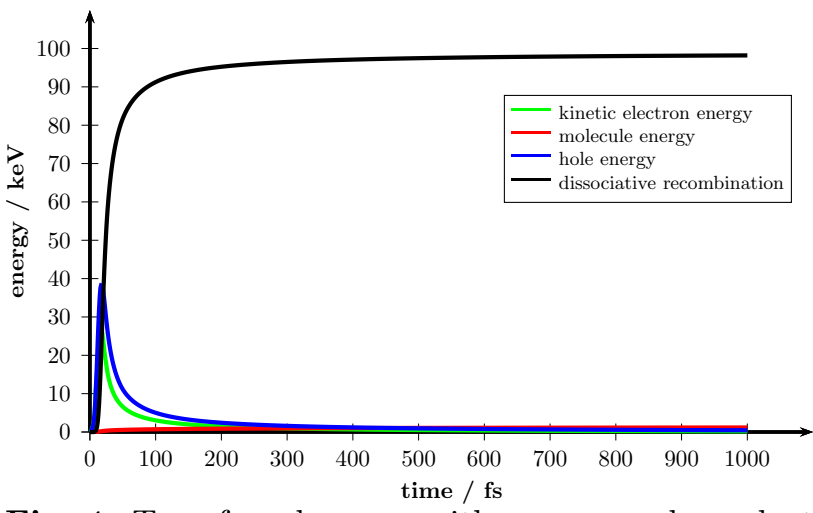

Fig. 4: Transferred energy with an energy dependent dissociative recombination

To further investigate the energy transfer with an energy dependent cross section for dissociative recombination, we derive the transfer rates for elastic scattering, ionization and dissociative recombination from Figure 4 . Figure 5 shows the energy transfer rate for elastic scattering and Figure 6 both the rates for ionization and dissociative recombination. 
At first, it can be seen that the energy transfer rate of elastic scattering, Figure 5, is several orders of magnitude lower than those shown in Figure 6. This is due to the fact, that only a small fraction of energy can be transferred via elastic scattering because of the huge difference in the mass of an electron and a water molecule, compare equation 7 .

Figure 6 shows that the ionization transfer rate reaches its maximum during the laser irradiation, where both, photon absorption and impact ionization, occur. This increases the degree of ionization and thus the cross section of dissociative recombination. As this process reaches its maximum rate, the energy transfer rate due to ionization has dropped to zero and further decreases. This is due to the fact that dissociative recombination reduces the energy stored in the holes via the recombination of $\mathrm{H}_{2} \mathrm{O}^{+}$and $\mathrm{e}^{-}$.

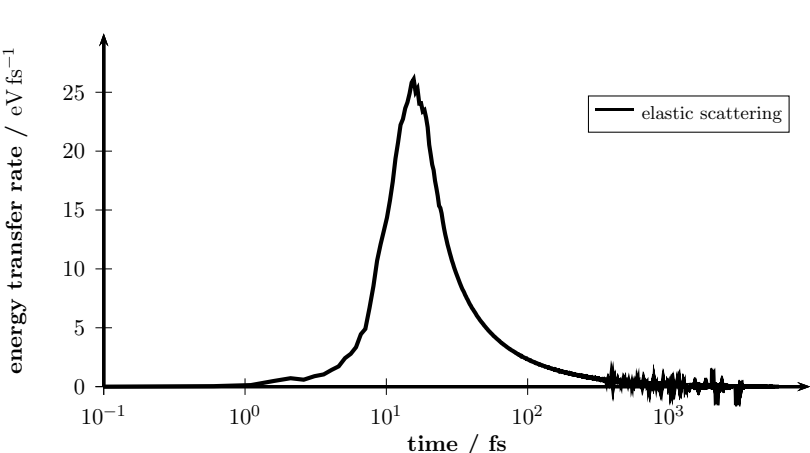

Fig. 5: Energy transfer rates of elastic scattering.

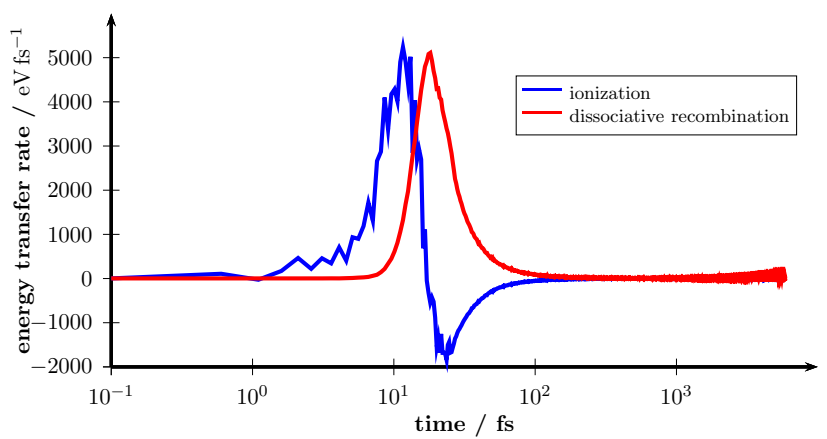

Fig. 6: Energy transfer rates of ionization and dissociative recombination.

\subsection{Band Gap: A first approach}

Here we present the results of our first approach to replace the distinctive molecule orbitals with a band structure of liquid water. According to Figure 2 in Ref. [10] we assume a distribution of energy levels in the region of $10 \mathrm{eV}$ to $20 \mathrm{eV}$ and $30 \mathrm{eV}$ to $37 \mathrm{eV}$. In our approach we consider a uniformly distributed density of states within these intervals. Thus, instead of creating an electron from one of the four molecule orbitals, the newly created electron must overcome a binding energy continuously distributed in the two energy intervals.

Figure 7 repeats Figure 2, calculated with molecule orbitals, to compare the course of the curve with the simulation containing a density of states as described above, see Figure 8. In Figure 8 mainly the curve after $10 \mathrm{fs}$ shows differences: The initial excitation peaks are now reproduced by two intervals and the number of electrons at low energies is slightly increased. In contrast, after $30 \mathrm{fs}$ a lower number of electrons is present as compared to Figure 7. Reasons for these differences and other mechanisms as the energy channels (similar to Figure 4) are currently under study.

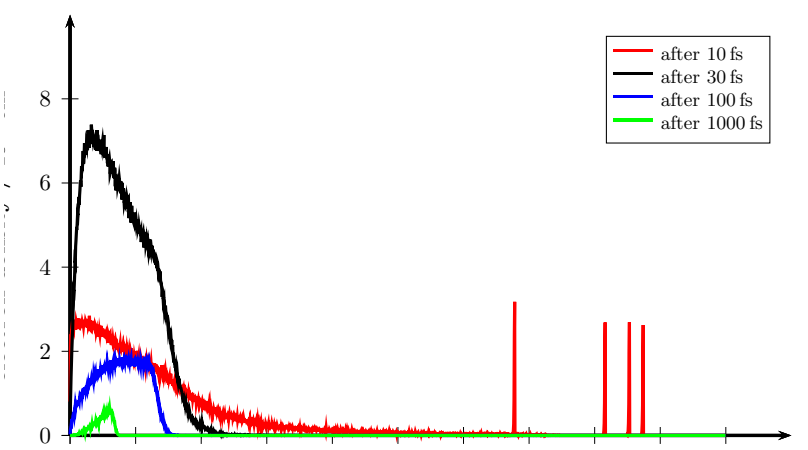

Fig. $7:^{10}$ Enerergy $^{30}$ resolved ${ }^{50}$ electron $^{60}$ density ${ }^{80}$ at certain time steps, containing distinctive molecule orbitals

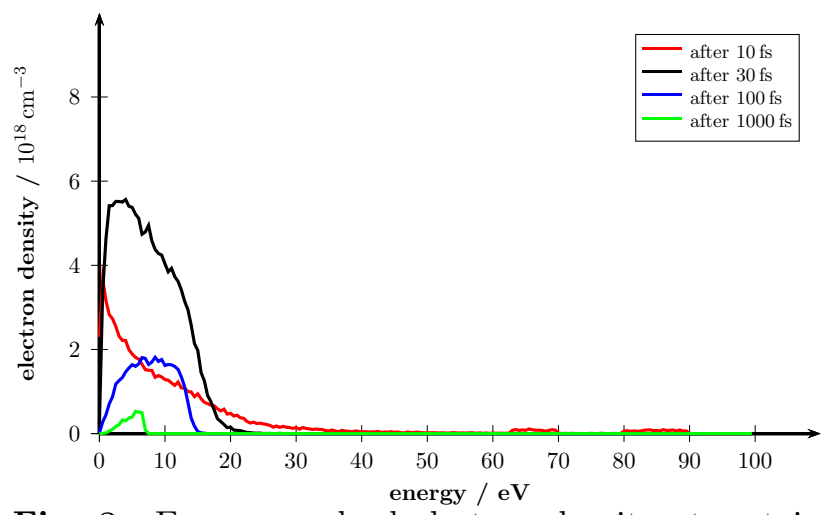

Fig. 8: Energy resolved electron density at certain time steps, containing a uniformly distributed density of states, see text 


\section{APPLICATION}

As an application we present the results for laser absorption in a thin ice layer of $60 \mathrm{~nm}$. As an approximation we treat the ice layer with our model assuming molecule orbitals and include the laser parameters as well as the average absorption depth of $31.3 \mathrm{~nm}$ for the wavelength of $13.5 \mathrm{~nm}$ [4].

Furthermore we simulate our control volume of $10^{3} \mathrm{~nm}^{3}$ at certain depths from $0 \mathrm{~nm}$ to $50 \mathrm{~nm}$ at the intervals of $10 \mathrm{~nm}$. According to the exponential absorption law, Figure 9 shows the time evolution of the density of the free electrons within the simulated volume. We assume, that more electrons are created beneath the surface than deeper in the layer. The number density increases rapidly during laser excitation and decreases slowly after the pulse is gone due to the energy dependent dissociative recombination.

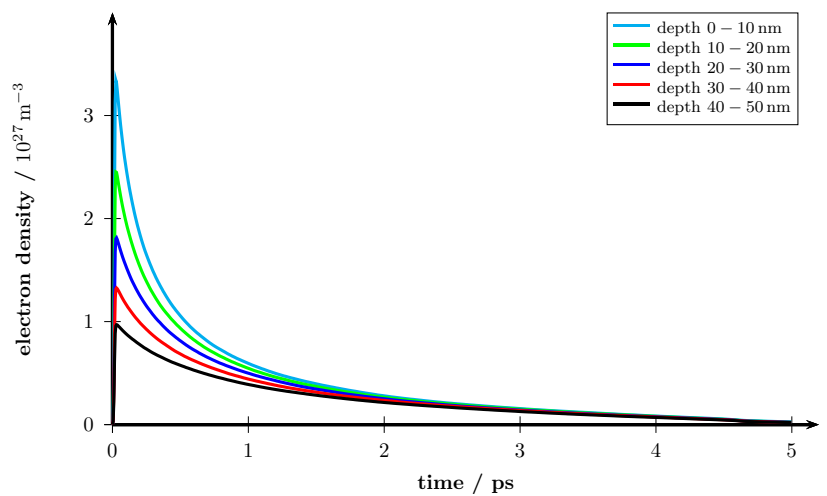

Fig. 9: This Figure shows the time resolved number density of the free electrons.

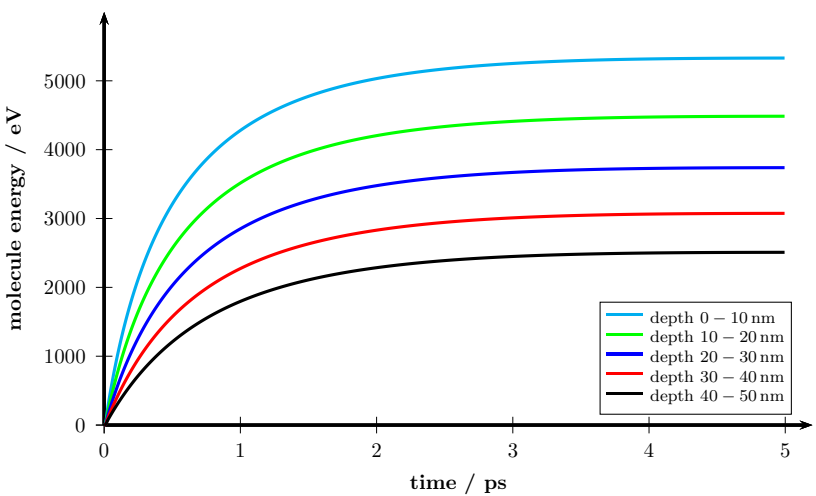

Fig. 10: Energy transfer from kinetic electron energy to the molecule energy via elastic scattering.

Furthermore we present the time resolved energy transfer from electrons to water molecules via elastic scattering, since the increasing energy of the molecules represents the heating of the probe. Figure 10 shows the time-resolved integrated transfer rate at certain depths. The integrated transfer rate follows the curve presented in section 3. Again, as more free electrons are created beneath the surface, the water molecules are more heated up in this region.

\section{SUMMARY}

In this work we showed and discussed the results of our model to simulate the electron dynamics in liquid water irradiated by an ultrashort laser pulse with the MC method. We presented the difference in using an artificial dissociative recombination as well as an energy dependent cross section derived from a data set in Ref. [9]. This difference is studied through the examples of the energy resolved electron density at distinctive time steps as well as time resolved integrated transfer rates. Furthermore we investigated the differential transfer rates of the involved processes, elastic scattering, impact ionization and dissociative recombination. In addition to that we showed our first approach to leave the four molecule orbitals and come up with a band structure, modelled as uniformly distributed density of states. Finally we presented a first application of our model to the irradiation of a thin ice layer.

\section{OUTLOOK}

The actual model shall be extended into several ways to consider possible applications. At first, we introduce a band gap instead of distinctive molecule orbitals derived from the water molecule. First results are shown in this work, further study of the energy dissipation into various channels is necessary. Furthermore the energy transfer rates will be used as input parameters for an MD simulation.

Moreover, electron-electron scattering will be included by an Ensemble Monte Carlo Method approach [11]. This new model may also be applied as the intermediate step of a pulse propagation (PP) simulation, see Ref. [12, 13]. Whereas the PP code simulates the interaction of light and the electronic system, our MC model will couple the electrons to the water molecules and the interaction among themselves. Thus calculated electron distribution 
of the PP code will be the input into our MC calculations and give back a time-step updated distribution. Finally, with the incorporation of the electron-electron scattering, electron transport shall be included in our model to come up with a time resolved electron transport during laser irradiation and afterwards on a femtosecond time scale.

\section{ACKNOWLEDGMENT}

We acknowledge financial support of the Deutsche Forschungsgemeinschaft (grant RE 1141/11-1), the BMBF (FSP 301-FLASH) and the Carl-Zeiss-Stiftung.

\section{References}

[1] W. Ackermann et al. "Operation of a free-electron laser from the extreme ultraviolet to the water window". In: Nature Photonics 1 (2007), pp. 336-342.

[2] N. Medvedev and B. Rethfeld. "Transient dynamics of the electronic subsystem of semiconductors irradiated with an ultrashort vacuum ultraviolet laser pulse". In: New Journal of Physics 12 (2010), p. 073037.

[3] N. Medvedev et al. "Short-Time Electron Dynamics in Aluminum Excited by Femtosecond Extreme Ultraviolet Radiation". In: Phys. Rev. Lett. 107 (2011), p. 165003.

[4] The Center for X-Ray Optics. URL: http://www.cxro.lbl.gov/.

[5] D. P. Landau and K. Binder. A Guide to Monte Carlo Simulations in Statistical Physics. Cambridge University Press, 2000.

[6] Ianik Plante and Francis A Cucinotta. "Cross sections for the interactions of $1 \mathrm{eV}-100 \mathrm{MeV}$ electrons in liquid water and application to Monte-Carlo simulation of HZE radiation tracks". In: New Journal of Physics 11 (2009), p. 063047.

[7] Yukikazu Itikawa. Molecular Processes in Plasmas: Collisions of Charged Particles with Molecules (Springer Series on Atomic, Optical, and Plasma Physics). Springer, 2007.

[8] N. Medvedev K. Huthmacher and B. Rethfeld. "Dynamics of electrons in liquid water excited with an ultrashort VUV laser pulse". In: Proceedings of SPIE 8077 (2011).

[9] S. Rosen et al. "Recombination of simple molecular ions studied in storage ring: dissociative recombination of $\mathrm{H}_{2} \mathrm{O}^{+"}$. In: Faraday Discuss. 115 (2000), pp. 295-302.

[10] P. Cabral do Couto, S. G. Estácio, and B. J. Costa Cabral. "The Kohn-Sham density of states and band gap of water: From small clusters to liquid water". In: The Journal of Chemical Physics 123 (2005), p. 054510.

[11] Martin Moko and Antónia Moková. "Ensemble Monte Carlo simulation of electron-electron scattering: Improvements of conventional methods". In: Phys. Rev. B 44 (1991).

[12] J. R. Gulley. "Modeling free-carrier absorption and avalanching by ultrashort laser pulses". In: Proceedings of SPIE 8190 (2011).

[13] Jeremy R. Gulley et al. "Interaction of ultrashort-laser pulses with induced undercritical plasmas in fused silica". In: Phys. Rev. A 85 (2012), p. 013808. 